\title{
CFTR dysfunction induces vascular endothelial growth factor synthesis in airway epithelium
}

\author{
Clémence Martin ${ }^{1,2,10}$, Nathalie Coolen ${ }^{1,2,10}$, Yongzheng Wu ${ }^{3,4}$, Guiti Thévenot ${ }^{1}$, \\ Lhousseine Touqui ${ }^{3,4}$, Virginie Prulière-Escabasse ${ }^{5,6}$, Jean-François Papon ${ }^{5,6}$, \\ André Coste ${ }^{5,6}$, Estelle Escudier ${ }^{7,8}$, Daniel J. Dusser ${ }^{1,2}$, Isabelle Fajac ${ }^{1,9}$ and \\ Pierre-Régis Burgel ${ }^{1,2}$
}

\begin{abstract}
Affiliations: 'Université Paris Descartes, Sorbonne Paris Cité, Paris, ${ }^{2}$ Service de Pneumologie, Hôpital Cochin, AP-HP, Paris, ${ }^{3}$ Unité de Défense Innée et Inflammation, Institut Pasteur, Paris, ${ }^{4}$ INSERM U874, Paris, ${ }^{5}$ Dept of Otorhinolaryngology, Hôpital Intercommunal de Créteil, Créteil, ${ }^{6}$ INSERM, U955, Créteil, ${ }^{7}$ Service de Génétique et d'Embryologie médicales, Hôpital Armand-Trousseau, AP-HP, Paris, ${ }^{8}$ Inserm U933, Paris, and ${ }^{9}$ Service de Physiologie et Explorations Fonctionnelles, Hôpital Cochin, AP-HP, Paris, France. ${ }^{10}$ These authors contributed equally to this work.
\end{abstract}

Correspondence: P-R. Burgel, Service de Pneumologie, Hôpital Cochin, AP-HP, 27, rue du Faubourg Saint Jacques, 75014 Paris, France. E-mail: pierre-regis.burgeldacch.aphp.fr

ABSTRACT Peribronchial angiogenesis may occur in cystic fibrosis and vascular endothelial growth factor (VEGF)-A regulates angiogenesis in airways.

Peribronchial vascularity and VEGF-A expression were examined using immunocytochemistry and morphometric analysis in lung sections obtained in 10 cystic fibrosis patients at transplantation versus 10 control nonsmokers, and in two strains of Cftr-deficient mice versus wild-type littermates. Airway epithelial NCI-H292 cells and primary cultures of noncystic fibrosis human airway epithelial cells were treated with cystic fibrosis transmembrane conductance regulator (CFTR) inhibitors (CFTR-inh ${ }^{172}$ or PPQ-102) or transfected with a CFTR small interfering (si)RNA with or without a selective epidermal growth factor receptor tyrosine kinase inhibitor. Concentrations of VEGF-A and phosphorylated epidermal growth factor receptor were measured by ELISA.

Peribronchial vascularity was increased in cystic fibrosis patients, but not in Cftr-deficient mice. VEGF-A immunostaining was localised to airway epithelium and was increased in cystic fibrosis patients and in Cftrdeficient mice. In cultured airway epithelial cells, treatment with CFTR inhibitors or transfection with CFTR siRNA induced a twofold increase in VEGF-A production. CFTR inhibitors triggered epidermal growth factor receptor phosphorylation that was required for VEGF-A synthesis.

Cystic fibrosis airways at transplantation showed increased peribronchial vascularity and epithelial VEGF-A expression. CFTR dysfunction triggered epithelial synthesis of VEGF-A, which may contribute to vascular remodelling.

@ERSpublications

CFTR dysfunction triggers epithelial synthesis of VEGF-A, which may contribute to vascular remodelling http://ow.ly/p7ebY

For editorial comments see page 1438.

This article has supplementary material available from www.erj.ersjournals.com

Received: Oct 142012 | Accepted after revision: March 052013 | First published online: March 212013

Support statement: This work was funded by grants from Chancellerie des Universités de Paris (Legs Poix), Association Vaincre la Mucoviscidose and Association Cardif.

Conflict of interest: Disclosures can be found alongside the online version of this article at www.erj.ersjournals.com

Copyright CERS 2013 


\section{Introduction}

Cystic fibrosis (CF) is a genetic disease caused by mutations in the cystic fibrosis transmembrane conductance regulator (CFTR) gene [1]. CFTR protein dysfunction results in abnormal ion transport across the airway epithelium [2], progressively leading to chronic lung disease [1, 3]. CF lung disease is characterised by airway structural abnormalities that include bronchiectasis with mucus plugging, subepithelial fibrosis, remodelling of airway epithelium and submucosal glands, and hyperplasia of airway smooth muscle [4-6].

The bronchial circulation has the ability to proliferate and peribronchial angiogenesis (the proliferation of new blood vessels from existing vessels) is increasingly recognised as an important component in chronic airway diseases $[7,8]$. Abnormalities in peribronchial blood vessels may contribute to immune responses via microvascular leakage and recruitment of inflammatory cells $[9,10]$. Increased peribronchial vascularity was reported in asthma and in chronic obstructive pulmonary disease [7, 8]. However, to the best of our knowledge, peribronchial vascularity has not been examined in CF pathological studies.

Vascular endothelial growth factor (VEGF)-A is a key angiogenic mediator in the airways [8]. BALUK et al. [11] have shown that selective expression of VEGF-A in mouse airway epithelium triggered tracheal angiogenesis. Previous studies in CF subjects have reported increased VEGF-A concentrations in serum [12-14], but little is known about VEGF-A expression in CF airways. KRENN et al. [15] found increased VEGF-A mRNA and protein in lung homogenates of CF subjects undergoing lung transplantation. By contrast, MEYER et al. [13] found no increase in VEGF-A levels in bronchoalveolar lavage fluid of adult CF subjects. Thus, the expression pattern and the cellular localisation of VEGF-A in CF airways remain to be established.

In the present study, we examined peribronchial vascularity and VEGF-A immunostaining in lung tissue sections from CF subjects versus control nonsmokers and in the lungs of two different strains of Cftrdeficient mice versus wild-type littermates. Because the airway epithelium appeared as the main site of VEGF-A expression in CF airways and in Cftr-deficient mice, we studied the effects of CFTR dysfunction on VEGF-A synthesis in cultured airway epithelial cells.

\section{Methods}

Human and mouse tissues

Peripheral human lung tissues (containing noncartilaginous airways) were obtained at transplantation from 10 nonsmoking CF adults and from 10 nonsmoking controls undergoing lung resection for peripheral lung cancer. Clinical characteristics of these patients are provided in online supplementary table S1. Lung tissue samples were fixed in $10 \%$ neutral buffered formalin by inflation-immersion and embedded in paraffin. To reduce the potential sampling bias related to irregular distribution of morphological abnormalities in CF patients, tissues were obtained from two to four blocks (depending on tissue availability), whose locations were chosen randomly. In control subjects, only one block was available for study.

Cftr ${ }^{\text {mlUNC }}\left(\mathrm{Cftr}^{-/-}\right)$mice, established by gene targeting [16], and their wild-type littermates $\left(\mathrm{Cftr}^{+/+}\right)$were obtained from the Centre de Distribution, de Typage et d'Archivage Animal (Orleans, France). Cftr ${ }^{\text {tmlEur }}$ $\left(F 508^{d e l / d e l}\right)$ mice [17] and their wild-type littermates $\left(F 508^{w t / w t}\right)$ mice were a kind gift from B. Scholte (Erasmus University, Rotterdam, the Netherlands). After weaning, all mice were provided with a commercial osmotic laxative (Movicol; Norgine, Harefield, UK) in the drinking water to prevent intestinal occlusion. 8- to 10-week-old mice were sacrificed and lungs were fixed in $4 \%$ formalin and embedded in paraffin. Studies were performed on five to seven animals per group. The study conformed to the Declaration of Helsinki and to all the rules of the local Committees on Human and on Animal Research. Informed consent was obtained from all patients.

\section{Immunohistochemical staining and quantitative morphometric analysis}

Immunohistochemical staining was performed on $5-\mu \mathrm{m}$ paraffin-embedded sections, as previously described [18]. The following primary antibodies were used: a polyclonal antibody to VEGF-A (A 20, 1:200; Santa Cruz Biotechnology Inc., Santa Cruz, CA, USA); a polyclonal antibody to the endothelial marker von Willebrand factor (vWF, 1:50; DakoCytomation, Carpinteria, CA, USA) to identify blood vessels. Antigen unmasking by incubation with protease from Streptomyces griseus (0.01\%; Sigma-Aldrich Company Ltd, Gillingham, UK) for 20 min was required to improve the quality of staining for vWF. Biotinylated antirabbit antibody (1:200; Vector Laboratories, Burlingame, CA, USA) was used for secondary antibody, and bound antibodies were visualised according to standard protocols for avidin-biotin-peroxidase complex method (Elite ABC kit; Vector Laboratories, Peterborough, UK). Tissue sections were counterstained with haematoxylin. Omission of primary antibody and incubation with irrelevant immunoglobulins were used as negative controls. 
Quantification of peribronchial vascularity and of VEGF-A epithelial immunostaining were performed by point counting, as previously described $[4,18,19]$. Volume occupied by VEGF-A positively stained cells in epithelium was expressed per volume of epithelium. Peribronchial vascularity was defined as the volume occupied by vWF-positive blood vessels in the peribronchial space (defined as the space between epithelial basement membrane and the base of alveolar attachments) per volume of peribronchial space. All analyses were performed on at least 20 randomly chosen high-magnification $(400 \times)$ photomicrographs obtained in two to four distant sections per patient or per mouse.

\section{Culture of human airway epithelial cells}

NCI-H292 human airway epithelial cells (HAECs), which express functional CFTR [20] and produce VEGF-A [18], were plated at $10^{5}$ cells $\cdot \mathrm{mL}^{-1}$ in 24-well plates (BD Falcon; BD Biosciences, San Jose, CA, USA), and grown in RPMI 1640 medium containing $10 \%$ fetal calf serum (FCS), penicillin $\left(100 \mathrm{U} \cdot \mathrm{mL}^{-1}\right)$, streptomycin $\left(100 \mu \mathrm{g} \cdot \mathrm{mL}^{-1}\right)$ and HEPES $(25 \mathrm{mM})$ at $37^{\circ} \mathrm{C}$ in a humidified $5 \%$ carbon dioxide waterjacketed incubator.

Primary cultures of HAECs were obtained from five non-CF patients undergoing surgery for nasal polyps, as previously described [21]. Cells were plated at $10^{6}$ cells $\cdot$ well $^{-1}$ in inserts $(12 \mathrm{~mm}$; Transwell, Costar, Cambridge, MA, USA) with 12-mm-diameter type IV collagen-coated (Sigma-Aldrich) polycarbonate microporous membranes and were incubated with $1 \mathrm{~mL}$ of DMEM/F12/antibiotics containing 2\% Ultroser G (Gibco BRL, Life Technologies, Gaithersberg, MD, USA). Cells were cultured at an air-liquid interface for 3 weeks, at a time when they show full differentiation with ciliated and mucous cells [22]. Previous studies have shown that these cells: 1) produce VEGF-A protein with a peak of production during the first week of culture, and a decreased production thereafter until day 21 of culture [21]; and 2) have no CFTR expression and function during the first week of culture, but express functional CFTR after 2 weeks of culture [23]. Thus, studies using CFTR inhibitors (see below) were performed at day 21 of culture.

\section{CFTR inhibition in HAECs}

Inhibition of CFTR-mediated ion transport was obtained by treating airway epithelial cells with selective CFTR inhibitors (CFTR-inh ${ }^{172}$ or PPQ-102; Calbiochem, Nottingham, UK) [24, 25], which have been reported to stabilise the CFTR channel in a closed state. In brief, NCI-H292 cells at $60-80 \%$ confluence were treated either with CFTR-inh ${ }^{172}\left(10^{-5} \mathrm{M}\right)$ or with PPQ-102 $\left(10^{-5} \mathrm{M}\right)$ diluted in dimethyl sulfoxide (DMSO), or with DMSO alone (Sigma) for $72 \mathrm{~h}$. Time of exposure and concentrations of inhibitors were selected based on previous literature [24-26]. In preliminary experiments, we found that CFTR inhibitors induced VEGF-A synthesis when cells were serum starved (no FCS) during the last $48 \mathrm{~h}$ of culture and when cells were cultured in the presence of FCS (data not shown); all subsequent experiments were performed in FCS-containing medium. Because HAECs were cultured at the air-liquid interface, and because CFTR is expressed on the apical side of epithelial cells, treatments were applied both at the basolateral level (in culture medium), and at the apical level by dropping $150 \mu \mathrm{L}$ of culture medium on the cell surface [26]. Culture media were changed daily. After $72 \mathrm{~h}$ of incubation either with CFTR inhibitors or with DMSO, cell culture media were collected and stored at $-80^{\circ} \mathrm{C}$. VEGF-A concentrations, corresponding to VEGF-A production in $24 \mathrm{~h}$, were measured by ELISA (see below).

Knockdown of CFTR expression was obtained by transfecting NCI-H292 cells with a specific CFTR small interfering (si)RNA (Sigma), and nontargeted siRNA was used as a negative control [27]. Transfections were performed using TransIT-siQUEST1, according to the manufacturer's instructions (Mirus, Madison, WI, USA) [27]. As reported in a previous manuscript by our group [27], CFTR siRNA resulted in a 60-70\% knockdown in CFTR expression, as examined by Western blotting (data not shown). $24 \mathrm{~h}$ after transfection, cells were rinsed three times with fresh culture medium and incubated for additional $24 \mathrm{~h}$ before harvesting culture media that was stored at $-80^{\circ} \mathrm{C}$.

In selected experiments, epithelial cells were incubated for $24 \mathrm{~h}$ with a selective epidermal growth factor receptor (EGFR) inhibitor (AG1478, $10^{-5} \mathrm{M}$; Calbiochem) or its inactive analogue (AG9, $10^{-5} \mathrm{M}$; Calbiochem) [28]. For assessing the effect of CFTR inhibition on EGFR activation, NCI-H292 cells were treated with CFTR-inh ${ }^{172}$ or DMSO alone for $15 \mathrm{~min}, 1 \mathrm{~h}, 3 \mathrm{~h}, 6 \mathrm{~h}, 8 \mathrm{~h}$ and $24 \mathrm{~h}$. At each time-point, cells were washed twice with sterile phosphate-buffered saline and cell lysates were obtained by solubilising cells at $10^{7}$ cells $\cdot \mathrm{mL}^{-1}$ in lysate buffer ( $1 \% \mathrm{NP}-40$ Alternative (Santa Cruz Biotechnology, Heidelberg, Germany), $20 \mathrm{mM}$ Tris at $\mathrm{pH}$ 8.0, $137 \mathrm{mM} \mathrm{NaCl}, 10 \%$ glycerol, $2 \mathrm{mM}$ EDTA, $1 \mathrm{mM}$ activated sodium orthovanadate, $10 \mu \mathrm{g} \cdot \mathrm{mL}^{-1}$ aprotinin and $10 \mu \mathrm{g} \cdot \mathrm{mL}^{-1}$ leupeptin). Samples were allowed to sit on ice for $15 \mathrm{~min}$ before storage at $-80^{\circ} \mathrm{C}$. 
Measurements of VEGF-A and phosphorylated EGFR protein concentrations

VEGF-A in cell culture supernatants and phosphorylated EGFR (EGFR-P) in cell lysates were measured by sandwich ELISA kits according to the manufacturer's instructions (DuoSet IC; R\&D Systems, Minneapolis, MN, USA). EGFR-P results were expressed as pg of EGFR-P per $\mu$ g of total protein. Protein concentrations in cell lysates were measured using a bicinchoninic acid-based protein assay kit (Pierce, Rockford, IL, USA). Each sample was measured in duplicate.

\section{Quantitative PCR for VEGF mRNA}

Total RNA was extracted from NCI-H292 cells using RNeasy mini kit (Qiagen, Hilden, Germany). $1 \mu \mathrm{g}$ of total RNA was used for reverse transcription following quantitative PCR (qPCR) amplification of VEGF-A and $\beta$-actin expression using 7900HT fast real-time PCR system (Applied Biosystems; Life Technologies Ltd, Paisley, UK). The qPCR primers were used as VEGF-A: 5'-AGGGCAGAATCATCACGAAGT-3' (forward), 5'-AGGGTCTCGATTGGATGGCA-3' (reverse), and as $\beta$-actin: 5'-GCAAACGTGCGTGACAT$3^{\prime}$ (forward), 5'-TCGCGACAGGTCTTTGC-3' (reverse). The relative quantification of gene expression was calculated using $\Delta \Delta \mathrm{Ct}$ method and results were presented as fold increase compared with the corresponding control at each time-point.

\section{Statistical analysis}

Data obtained from morphometric analysis were presented as $\%$ and were analysed using the nonparametric Mann-Whitney U-test and the Spearman's rank correlation. The interobserver coefficients of variation for morphometric measurements were $<15 \%$. Data obtained from cell culture experiments were presented as mean \pm SEM of at least three independent experiments performed in duplicate and were analysed using oneway ANOVA for repeated measurements, followed by the post hoc Student-Neuman-Keuls test for multiple comparisons. All analyses were performed using the Prism 5 software (GraphPad Software, Inc., San Diego, CA, USA). p-values $\leqslant 0.05$ were considered to indicate statistical significance.

\section{Results}

Peribronchial vascularity and VEGF-A immunostaining in human and mouse airways

In control nonsmokers, the peribronchial space was thin and immunostaining for vWF identified sparse peribronchial blood vessels. In CF subjects, the peribronchial space was markedly thickened and contained numerous blood vessels. Morphometric analysis showed that peribronchial vascularity was increased by twofold in CF airways $(\mathrm{p}<0.01$ ) (fig. 1a). Immunostaining for VEGF-A was positive in lung sections of all control and CF subjects. Staining for VEGF-A was predominantly localised to the airway epithelium, where it was present in ciliated, but not in goblet cells. In CF airways, VEGF-A staining was also present in macrophages, but not in neutrophils (not shown). Morphometric analysis showed that VEGF-A staining was markedly increased in the airway epithelium of CF subjects $(p<0.05)$ (fig. 1b). When grouping both controls and CF subjects, a strong correlation between epithelial VEGF-A-stained volume density and peribronchial vascular volume density was found $(\mathrm{r}=0.65 ; \mathrm{p}<0.05)$ (fig. $1 \mathrm{c})$. However, this correlation was not significant when limiting the analysis to CF subjects $(\mathrm{r}=0.45 ; \mathrm{p}=0.19)$.

In $\mathrm{Cftr}^{-/-}$and $\mathrm{F} 508^{\mathrm{del} / \mathrm{del}}$ mice and in wild-type mice, immunostaining for VEGF-A was also localised to airway epithelium. Morphometric analysis showed increased VEGF-A-staining in the epithelium of $\mathrm{Cftr}^{-/-}$ and F508 ${ }^{\text {del/del }}$ mice compared with their respective wild-type littermates (fig. 2). No difference in peribronchial vascularity was found when comparing Cftr-deficient and wild-type mice (data not shown).

Representative photomicrographs of immunostaining for VEGF-A in humans and in mice are shown in figure 3. Representative photomicrographs of vWF immunostaining in human subjects are presented in online supplementary figure S1.

Effect of CFTR inhibition on VEGF-A synthesis in cultured human airway epithelial NCl-H292 cells Measurement of short-circuit current confirmed that NCI-H292 cells had chloride transport that was inhibited by the selective CFTR inhibitors, CFTR-inh ${ }^{172}$ or PPQ-102 (see online supplementary fig. 2). Treatment of NCI-H292 cells with CFTR-inh ${ }^{172}\left(10^{-5} \mathrm{M}\right)$ or with PPQ-102 $\left(10^{-5} \mathrm{M}\right)$ induced a twofold increase in VEGF-A synthesis ( $\mathrm{p}<0.05$ versus baseline) (fig. 4a). Similarly, transfection of NCI-H292 cells with a specific CFTR siRNA increased VEGF-A synthesis $(\mathrm{p}<0.05$ versus baseline) (fig. $4 \mathrm{~b})$, whereas transfection with a nontargeted siRNA had no effect.

To examine whether the effect of CFTR inhibition on VEGF-A synthesis was related to a transcriptional effect, we examined VEGF-A mRNA expression using qPCR. Treatment of NCI-H292 cells with CFTR$\operatorname{inh}^{172}\left(10^{-5} \mathrm{M}\right)$ induced increased VEGF-A mRNA at $12 \mathrm{~h}$, peaked at $24 \mathrm{~h}$ and then decreased (see fig. $4 \mathrm{c}$ ). 

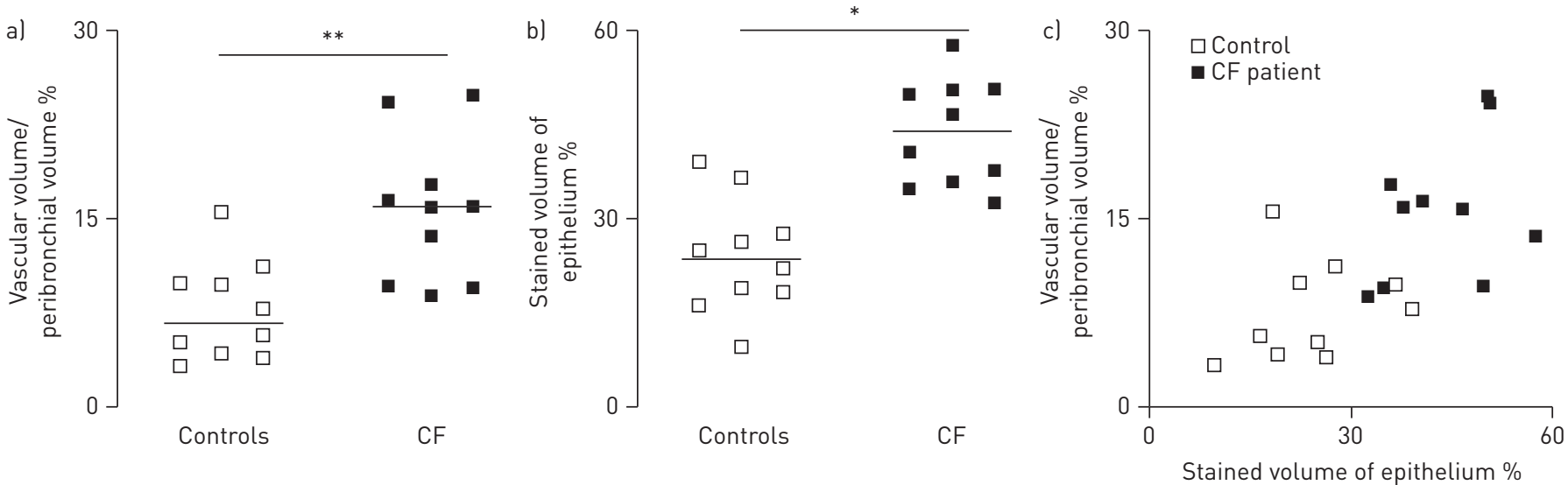

FIGURE 1 Morphometric analysis of peribronchial vascularity and vascular endothelial growth factor (VEGF)-A immunostaining in the airways of controls $(n=10)$ and of cystic fibrosis $(C F)$ subjects $(n=10)$. Airway sections were immunostained for the endothelial marker von Willebrand factor (to identify blood vessels) or for VEGF-A. Morphometric analysis was performed using point counting (see Methods section). a) Shows the peribronchial vascularity, defined as the volume occupied by blood vessels (vascular volume) in the peribronchial space/volume of peribronchial space (\%). b) Shows the volume of epithelium positively stained for VEGF-A as a percentage. c) Shows the vascular volume/peribronchial volume as a percentage and the volume of epithelium stained positively for VEGF-A as a percentage. Correlation between VEGF-A in epithelium and peribronchial vascularity was performed using the nonparametric Spearman's rank correlation. This correlation was highly significant $(\mathrm{r}=0.65 ; \mathrm{p}<0.05)$ when considering both control and $\mathrm{CF}$ subjects, but was not significant when considering only CF subjects $(r=0.45 ; \mathrm{p}=0.19)$. Horizontal bars represent medians. ${ }^{*}: \mathrm{p}<0.05 ;{ }^{*}$ : $\mathrm{p}<0.01$.

Role of EGFR phosphorylation in VEGF-A synthesis induced by CFTR inhibition in NCl-H292 cells Because EGFR phosphorylation is required for VEGF-A synthesis, which is induced by various stimuli in airway epithelial cells [18, 29], we examined its role in VEGF-A synthesis induced by CFTR inhibition. Treatment of NCI-H292 cells with CFTR-inh ${ }^{172}$ induced a significant increase in EGFR phosphorylation at $8 \mathrm{~h}\left(\mathrm{p}<0.05\right.$ versus vehicle, and $\mathrm{p}<0.05$ versus CFTR-inh ${ }^{172}$ at $\left.15 \mathrm{~min}\right)$ (fig. $\left.4 \mathrm{~d}\right)$. Pretreatment of NCI-H292 cells with AG1478 $\left(10^{-5} \mathrm{M}\right.$, a selective inhibitor of EGFR tyrosine kinase) prevented VEGF-A synthesis induced by CFTR chemical inhibitors or by CFTR siRNA $(\mathrm{p}<0.05$ versus baseline) (fig. $4 \mathrm{a}$ and $\mathrm{b})$. Pretreatment of NCI-H292 cells with AG9 $\left(10^{-5} \mathrm{M}\right.$, an inactive analogue of AG1478) was without effect.

Role of functional CFTR in VEGF-A synthesis in primary cultures of HAECs

Primary cultures of HAEC isolated from freshly excised nasal polyps secreted VEGF-A during the first week of culture, which gradually decreased over time, confirming previously published data (see online supplementary fig. S3) [21]. At day 21 of culture, differentiated HAECs produced low levels of VEGF-A, but treatment with CFTR-inh ${ }^{172}$ increased VEGF-A synthesis, which was prevented by AG1478 $(\mathrm{p}<0.05)$ (fig. 4e).

\section{Discussion}

We found that the airway epithelium was the main site of VEGF-A immunostaining in the lung of CF subjects obtained at transplantation, and that VEGF-A expression was increased in CF as compared with control subjects. Immunostaining for VEGF-A was also increased in the airway epithelium in two different mouse strains with CFTR dysfunction. In cultured airway epithelial cells, inhibition of CFTR (using chemical inhibitors or siRNA) resulted in increased VEGF-A synthesis, which required EGFR activation. These data suggest that CFTR dysfunction contributes to VEGF-A upregulation in the airway epithelium of CF subjects. They further suggest roles for epithelial VEGF-A in the peribronchial vascular remodelling that occurs in CF airways.

We report for the first time increased expression of VEGF-A in CF airway epithelium. MCCOLLEY et al. [12] reported increased serum VEGF-A levels in stable CF subjects versus subjects with non-CF pulmonary diseases, but VEGF-A levels were not measured in the airway compartment. MEYER et al. [13] measured similar concentrations of VEGF-A in the bronchoalveolar lavage fluid of nine CF and 16 age-matched healthy volunteers. Because VEGF-A was degraded in vitro by CF bronchoalveolar lavage fluid, which contained high neutrophil elastase activity, these authors were unable to derive a definitive conclusion on pulmonary production of VEGF-A in CF subjects [13]. KRENN et al. [15] found increased VEGF mRNA and protein in lung homogenates of CF subjects at transplantation as compared with lungs obtained in nonsmokers. Our data confirm these latter findings and extend these data by showing that the airway epithelium is the main site of VEGF-A expression in CF lungs. 


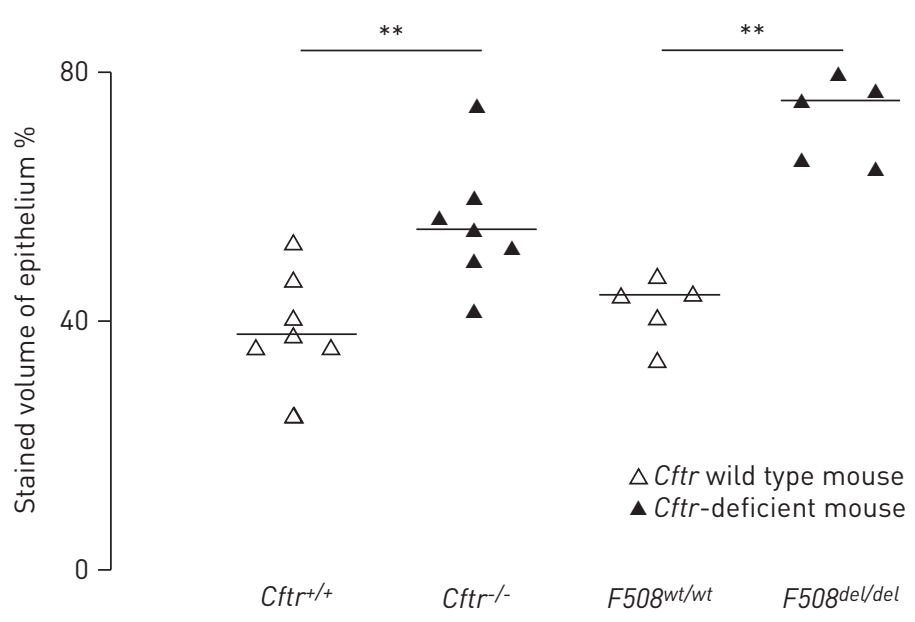

FIGURE 2 Morphometric analysis of vascular endothelial growth factor (VEGF)-A immunostaining in the airway epithelium of cystic fibrosis transmembrane conductance regulator ( $C f t r)$-deficient and wildtype mice. Airway sections were obtained in Cftr ${ }^{\text {mIUNC }}\left(\mathrm{Cftr}^{-1-}\right)$ mice and their wild-type $\left(\mathrm{Cftr}^{+/+}\right)$ littermates (left), and in Cftr ${ }^{\text {tm1Eur }}$ $\left(F 508^{d e l / d e l}\right)$ and their wild-type $\left(F 508^{w t / w t}\right)$ littermates (right). Sections were immunostained for VEGF-A and morphometric analysis was performed as described in the Methods section. Horizontal bars represent medians. ${ }^{* *} \mathrm{p}<0.01$.

Our conclusion that CFTR dysfunction triggers VEGF-A synthesis is based on data obtained in cultured airway epithelial cells. Previously, VerHAEGHE et al. [30] reported increased VEGF-A synthesis in a human fetal cell line carrying the homozygous mutation F508del compared with a control human fetal cell line. These latter data provided circumstantial evidence of a role for CFTR dysfunction in VEGF-A production; however, differences between these two cell lines could have been unrelated to CFTR dysfunction [31]. In the present study, inhibition of CFTR channel function using chemical inhibitors, or knockdown of CFTR using CFTR siRNA in a non-CF cell line and in non-CF HAECs provided direct evidence that CFTR dysfunction triggered VEGF-A synthesis. Increased immunostaining for VEGF-A in the airway epithelium of Cftr-deficient mice provided further evidence for this finding.

The finding that increased VEGF-A expression was not associated with increased peribronchial vascularity in Cftr-deficient mice was somewhat surprising. Because VEGF-A stimulates endothelial cell proliferation and angiogenesis via its effects on vascular endothelial growth factor receptor 2 (VEGFR2) [32], we examined the expression of VEGFR2 in mouse lung. In wild-type and in Cftr-deficient (both Cftr ${ }^{-1}$ and F508 ${ }^{\text {del/del }}$ ) mice, immunostaining for VEGFR2 was localised in the airway epithelium, but was absent in the endothelium of peribronchial blood vessels (see online supplementary fig. S4). In contrast, BALUK et al. [11] found that VEGF-A overexpression in airway epithelium, which resulted with tracheal angiogenesis, was associated with expression of VEGFR2 in the endothelium of tracheal blood vessels. We suggest that the lack of VEGFR2 expression in endothelium may have accounted for the absence of increased vascularity in Cftrdeficient mice.

The strength of our study relies on the use of complementary models and sources of information, including human CF tissues, mouse models and cultured HAECs. We also recognise limitations to our approaches. The correlation that was found between VEGF-A in epithelium and peribronchial vascularity was significant only when grouping controls and CF subjects. The lack of correlation between peribronchial vascularity and epithelial VEGF-A in CF subjects may be related to the relatively small number of CF tissues available, and to the homogeneous characteristics of these patients who were recruited at transplantation. Alternatively, epithelial VEGF-A may not be associated with peribronchial angiogenesis in CF subjects and may play different roles (e.g. epithelial proliferation [33]). CF subjects at transplantation were younger than control subjects. Because a previous study of VEGF-A immunostaining in young adults with normal lung function reported no immunostaining in the airway epithelium [34], this difference was unlikely to affect our findings. Mouse models of CFTR dysfunction do not reproduce the structural abnormalities typical of human CF lung disease [35]. Thus, the absence of increased peribronchial vascularity in Cftr-deficient mice should be interpreted cautiously. However, one might hypothesise that besides CFTR dysfunction, other factors inducing VEGF-A expression, such as bacterial infection [18], might be necessary to promote peribronchial angiogenesis. Finally, CFTR inhibition induced both increased VEGF-A mRNA and protein synthesis, suggesting a transcriptional effect. However, further studies will be necessary to determine whether the effect is related to mRNA synthesis or mRNA stabilisation.

Our findings indicated that CFTR dysfunction triggered VEGF-A synthesis in airway epithelium, independently of infection and inflammation. However, increased VEGF-A expression that was present in $\mathrm{CF}$ airway epithelium at transplantation could have been related to multiple factors: pro-inflammatory cytokines (e.g. tumour necrosis factor- $\alpha$ and interleukin-1 $\beta$ ), microbial products from Gram-negative and 

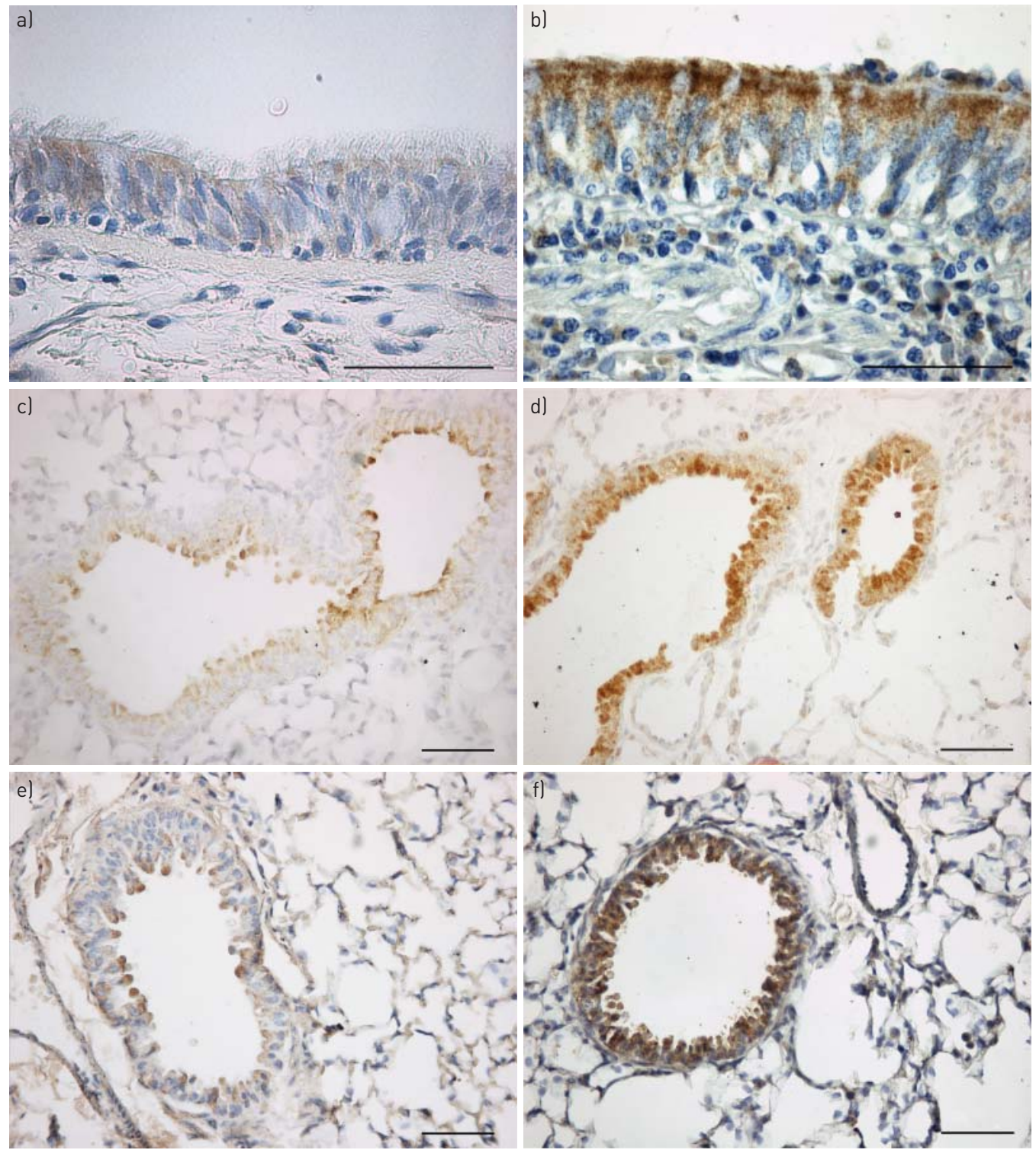

FIGURE 3 Representative photomicrographs of vascular endothelial growth factor (VEGF)-A immunostaining in human and in mouse airways. Airway sections were obtained in a) human control patients versus b) cystic fibrosis (CF) patients, in c) cystic fibrosis transmembrane conductance regulator $(\mathrm{Cftr})^{+/+}$versus d) $\mathrm{Cftr}^{-1-}$ mice and in e) $\mathrm{F}_{0} 08^{\text {wt/wt }}$ versus $\mathrm{f}$ ) F508 del/del mice. Sections were stained with an antibody to VEGF-A (brown colour) and counterstained with haematoxylin. In control patients and in $\mathrm{Cftr}^{+++}$and $\mathrm{F} 508^{w t / w t}$ mice, only sparse staining for VEGF-A was present in the epithelium. In CF patients,

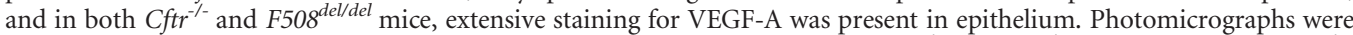
representative of results obtained in 10 controls and in $10 \mathrm{CF}$ patients, in seven $\mathrm{Cftr}^{+/+}$and $\mathrm{Cftr}^{-/-}$mice, and in five $\mathrm{F}^{2} 8^{\mathrm{wt} / \mathrm{wt}}$ and $F 508^{\text {delldel }}$ mice. Scale bars $=50 \mu \mathrm{m}$.

Gram-positive bacteria [18, 29] and viruses [29], and hypoxia [36] have also been reported to increase VEGF-A synthesis in airway epithelium.

VEGF-A was localised to ciliated cells, probably as a result of EGFR activation in ciliated cells or in precursors of ciliated cells (e.g. basal cells). Other major products of EGFR activation in the airway epithelium (e.g. mucins and interleukin-8) have been localised in secretory cells [4]. The localisation of EGFR in airway epithelial cell subpopulation is somewhat controversial in the literature: TYNER et al. [37] reported selective expression of EGFR in ciliated cells in vivo and in cultured epithelial cells, and suggested transdifferentiation of ciliated cells into mucin producing cells. Others have found EGFR expression in basal cells and in nongranulated secretory cells, but not in ciliated cells $[4,38]$. It is suggested that EGFR localisation is a dynamic process that may vary under various pathophysiological conditions. 

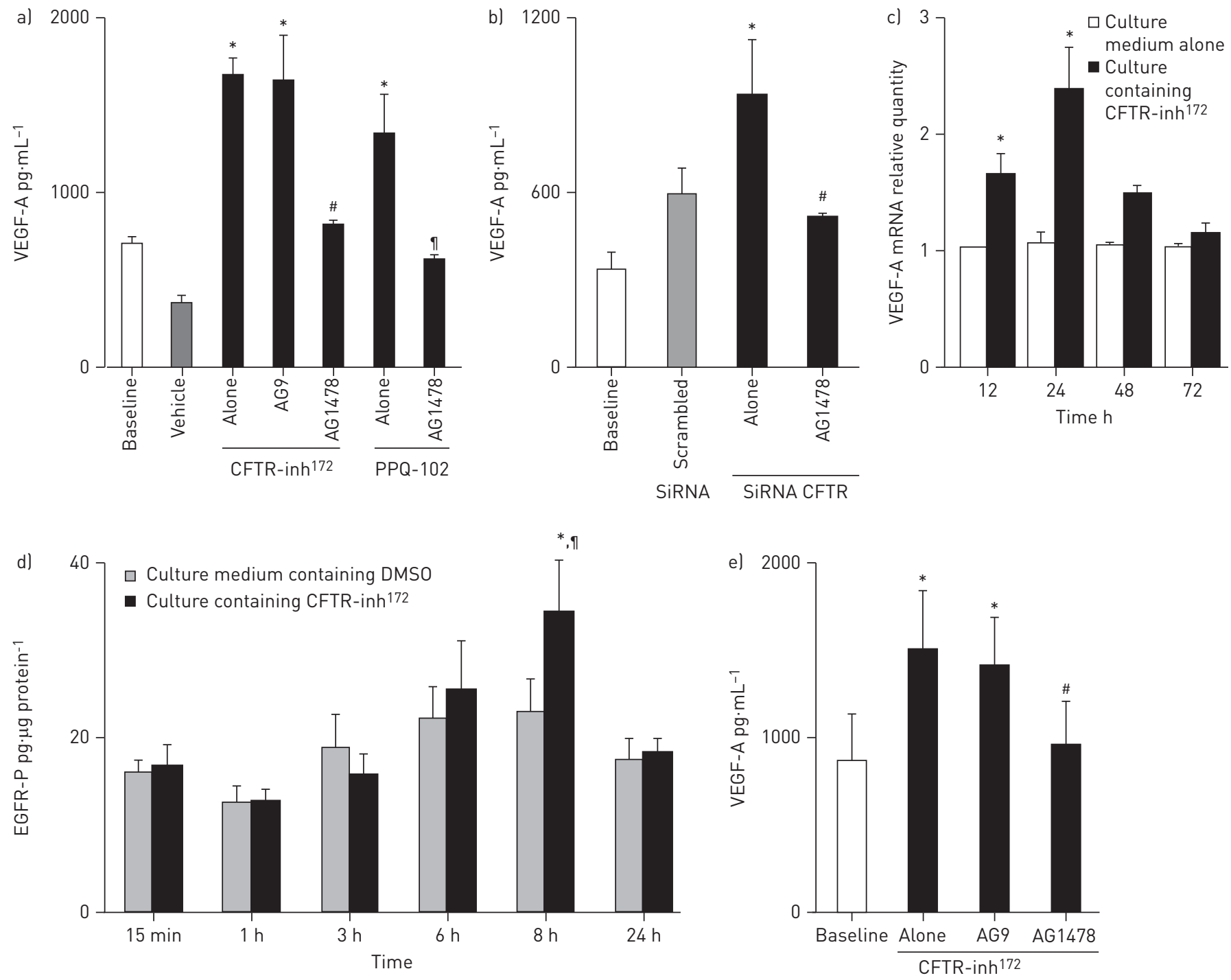

FIGURE 4 Effect of cystic fibrosis transmembrane conductance regulator (CFTR) inhibition on vascular angiogenic growth factor (VEGF)-A synthesis and epidermal growth factor receptor (EGFR) activation in airway epithelial cells. a) Effect of selective CFTR inhibitors on VEGF-A synthesis in NCI-H292 cells: Cells were cultured in culture medium alone (baseline), culture medium containing dimethyl sulfoxide (vehicle) or culture medium containing selective CFTR inhibitors (CFTR-inh ${ }^{172}$ or PPQ-102; $10^{-5} \mathrm{M}$ ). VEGF-A concentrations were measured by ELISA (see Methods section). In some experiments, cells were pretreated with the selective EGFR tyrosine kinase inhibitor AG1478 $\left(10^{-5} \mathrm{M}\right)$ or with its inactive analogue AG9 $\left(10^{-5} \mathrm{M}\right)$. Results were expressed as mean + SEM of $\mathrm{n} \geqslant 3$ independent experiments in duplicate. ${ }^{*}: \mathrm{p}<0.05$ versus baseline; ${ }^{*}: \mathrm{p}<0.05$ versus CFTR-inh ${ }^{172}$ alone; ${ }^{\bullet} \mathrm{p}<0.05$ versus $\mathrm{PPQ}-102$ alone. $\left.\mathrm{b}\right)$ Effect of transfection with a CFTR small interfering (si)RNA on VEGF-A synthesis in NCI-H292 cells. Cells were cultured in cell culture medium alone (baseline), culture medium transfected with a nontargeted (scrambled) siRNA or culture medium with a specific CFTR siRNA (see Methods section). Results were expressed as mean \pm SEM, $n \geqslant 3$ independent experiments in duplicate. ${ }^{*}: \mathrm{p}<0.05$ versus baseline; ${ }^{\#}: \mathrm{p}<0.05$ versus CFTR siRNA alone. c) Effect of CFTR-inh ${ }^{172}$ on VEGF-A mRNA expression in NCI-H292 cells. Cells were cultured in culture medium alone (baseline) or culture medium containing CFTR-inh ${ }^{172}$ (10 ${ }^{-5}$ M). VEGF mRNA was quantified using quantitative PCR and reported as relative quantity. $n=3$ independent experiments in duplicates. ${ }^{*}: \mathrm{p}<0.05$ compared with baseline at each time-point. d) Effect of CFTR-inh ${ }^{172}$ on EGFR phosphorylation in NCI-H292 cells. NCI-H292 cells were cultured in culture medium containing dimethyl sulfoxide (DMSO) or culture medium containing CFTR-inh ${ }^{172}\left(10^{-5} \mathrm{M}\right)$. Cell lysates were collected at different time-points, and phosphorylated EGFR (EGFR-P) concentrations were measured by ELISA (see Methods sections). Results were expressed as mean \pm SEM from $\mathrm{n} \geqslant 3$ independent experiments in duplicate. ${ }^{*}: \mathrm{p}<0.05$ versus DMSO at $8 \mathrm{~h} ;{ }^{\circ}: \mathrm{p}<0.01$ versus CFTR-inh ${ }^{172}$ at 15 min. e) Effect of CFTR-inh ${ }^{172}$ on VEGF-A synthesis in primary culture of human airway epithelial cells (HAECs) at the air-liquid interface. At day 21 of culture, HAECs were cultured in culture medium alone (baseline) or culture medium containing CFTR-inh ${ }^{172}\left(10^{-5} \mathrm{M}\right)$ for $72 \mathrm{~h}$. Results were expressed as mean \pm SEM from $\mathrm{n}=5$ independent experiments in duplicate. ${ }^{*}: \mathrm{p}<0.05$ versus baseline; ${ }^{*}: \mathrm{p}<0.05$ versus CFTR-inh ${ }^{172}$ alone.

Inhibition of CFTR function using CFTR-inh ${ }^{172}$ triggered EGFR phosphorylation, and increased VEGF synthesis after CFTR inhibition required EGFR activation in airway epithelial cells. Treatment of NCI-H292 cells with CFTR-inh ${ }^{172}$ resulted in the inhibition of chloride transport within minutes, confirming previous findings by PEREZ et al. [26], but EGFR phosphorylation was increased at $8 \mathrm{~h}$. Because activation of EGFR by its ligands (e.g. epidermal growth factor (EGF) or transforming growth factor- $\alpha$ ) usually occurs within seconds, this data indicates that CFTR-inh ${ }^{172}$ is not an EGFR ligand and suggests that activation of EGFR 
under these circumstances occurs via a cascade of events in epithelial cells. The precise molecular mechanisms leading to EGFR activation remain to be established.

Increased VEGF-A synthesis in airway epithelium could contribute to angiogenesis $[11,30]$ and increase in vascular permeability, facilitating recruitment of inflammatory cells (e.g. neutrophils) and proteins in the airways. Further, peribronchial angiogenesis could contribute to the occurrence of massive haemoptysis, a potentially life-threatening complication in CF patients [39]. Massive haemoptysis in CF patients is usually treated by bronchial artery embolisation. High rates of recurrence are observed after embolisation in these patients, presumably related to ongoing peribronchial angiogenesis and inflammation. Recurrent haemoptysis uncontrolled by repeated embolisation is a recognised criterion for lung transplantation in CF patients. We suggest that therapies directly targeting angiogenesis may be useful in CF patients with recurrent haemoptysis, but further studies will be necessary before such therapies may be considered in humans.

In summary, VEGF-A expression was markedly increased in CF airway epithelium at transplantation, suggesting roles in peribronchial angiogenesis in CF patients [11]. CFTR dysfunction directly contributed to VEGF-A upregulation in airway epithelium via EGFR activation. Therapies directly targeting CFTR dysfunction [40, 41] may result in significant reduction of VEGF-A synthesis and of peribronchial vascularity. Additionally, growth factor (e.g. EGF and/or VEGF) receptors antagonists could represent a promising way for reducing vascular remodelling in CF airways.

\section{Acknowledgements}

The authors thank Sophie Danel-Beaucaire (Cochin Hospital, Paris, France) for help with immunohistochemical staining and Claire Danel (Bichat Hospital, Paris, France) for providing human CF tissues.

\section{References}

O'Sullivan BP, Freedman SD. Cystic fibrosis. Lancet 2009; 373: 1891-1904.

Cohen TS, Prince A. Cystic fibrosis: a mucosal immunodeficiency syndrome. Nat Med 2012; 18: 509-519.

Cohen-Cymberknoh M, Shoseyov D, Kerem E. Managing cystic fibrosis strategies that increase life expectancy and improve quality of life. Am J Respir Crit Care Med 2011; 183: 1463-1471.

4 Burgel PR, Montani D, Danel C, et al. A morphometric study of mucins and small airway plugging in cystic fibrosis. Thorax 2007; 62: 153-161.

5 Hays SR, Ferrando RE, Carter R, et al. Structural changes to airway smooth muscle in cystic fibrosis. Thorax 2005; 60: $226-228$

6 Regamey N, Jeffery PK, Alton EW, et al. Airway remodelling and its relationship to inflammation in cystic fibrosis. Thorax 2011; 66: 624-629.

7 McCullagh A, Rosenthal M, Wanner A, et al. The bronchial circulation - worth a closer look: a review of the relationship between the bronchial vasculature and airway inflammation. Pediatr Pulmonol 2010; 45: 1-13.

8 Paredi P, Barnes PJ. The airway vasculature: recent advances and clinical implications. Thorax 2009; 64: 444-450.

9 Danese S, Dejana E, Fiocchi C. Immune regulation by microvascular endothelial cells: directing innate and adaptive immunity, coagulation, and inflammation. J Immunol 2007; 178: 6017-6022.

10 Frantz S, Vincent KA, Feron O, et al. Innate immunity and angiogenesis. Circ Res 2005; 96: 15-26.

11 Baluk P, Lee CG, Link H, et al. Regulated angiogenesis and vascular regression in mice overexpressing vascular endothelial factor in airways. Am J Pathol 2004; 165: 1071-1085.

12 McColley SA, Stellmach V, Boas SR, et al. Serum vascular endothelial growth factor is elevated in cystic fibrosis and decreases with treatment of acute pulmonary exacerbation. Am J Respir Crit Care Med 2000; 161: 1877-1880.

13 Meyer KC, Cardoni A, Xiang ZZ. Vasular endothelial growth factor in bronchoalveolar lavage from normal subjects and patients with diffuse parenchymal lung disease. J Lab Clin Med 2000; 135: 332-338.

14 Watts KD, McColley SA. Elevated vascular endothelial growth factor is correlated with elevated erythropoietin in stable young cystic fibrosis patients. Pediatr Pulmonol 2011; 46: 683-687.

15 Krenn K, Klepetko W, Taghavi S, et al. Vascular endothelial growth factor increases pulmonary vascular permeability in cystic fibrosis patients undergoing lung transplantation. Eur J Cardiothorac Surg 2007; 32: 35-41.

16 Snouwaert JN, Brigman KK, Latour AM, et al. An animal model for cystic fibrosis made by gene targeting. Science 1992; 257: 1083-1088.

17 French PJ, van Doorninck JH, Peters $\mathrm{RH}$, et al. A $\Delta \mathrm{F} 508$ mutation in mouse cystic fibrosis transmembrane conductance regulator results in a temperature-sensitive processing defect in vivo. J Clin Invest 1996; 98: 1304-1312.

18 Martin C, Thévenot G, Danel S, et al. Pseudomonas aeruginosa induces vascular endothelial growth factor synthesis in airway epithelium in vitro and in vivo. Eur Respir J 2011; 38: 939-946.

19 Hsia CC, Hyde DM, Ochs M, et al. An official research policy statement of the American Thoracic Society/ European Respiratory Society: standards for quantitative assessment of lung structure. Am J Respir Crit Care Med 2010; 181: 394-418.

20 Nagayama S, Kai H, Okiyoneda T, et al. Characterization of CFTR expression in a human pulmonary mucoepidermoid carcinoma cell line, NCI-H292 cells. FEBS lett 1999; 455: 215-218.

21 Coste A, Brugel L, Maitre B, et al. Inflammatory cells as well as epithelial cells in nasal polyps express vascular endothelial growth factor. Eur Respir J 2000; 15: 367-372.

22 Papon JF, Coste A, Gendron MC, et al. HLA-DR and ICAM-1 expression and modulation in epithelial cells from nasal polyps. Laryngoscope 2002; 112: 2067-2075.

23 Prulière-Escabasse V, Fanen P, Dazy AC, et al. TGF-beta 1 downregulates CFTR expression and function in nasal polyps of non-CF patients. Am J Physiol Lung Cell Mol Physiol 2005; 288: L77-L83.

24 Ma T, Thiagarajah JR, Yang H, et al. Thiazolidinone CFTR inhibitor identified by high-throughput screening blocks cholera toxin-induced intestinal fluid secretion. J Clin Invest 2002; 110: 1651-1658. 

inhibitor reduces cyst size in a polycystic kidney disease model. J Med Chem 2009; 52: 6447-6455

26 Perez A, Issler AC, Cotton CU, et al. CFTR inhibition mimics the cystic fibrosis inflammatory profile. Am J Physiol Lung Cell Mol Physiol 2007; 292: L383-L395.

27 Dif F, Wu YZ, Burgel PR, et al. Critical role of cytosolic phospholipase A2 $\alpha$ in bronchial mucus hypersecretion in CFTR-deficient mice. Eur Respir J 2010; 36: 1120-1130.

28 Burgel PR, Lazarus SC, Tam DCW, et al. Human eosinophils induce mucin production in airway epithelial cells via epidermal growth factor activation. J Immunol 2001; 167: 5948-5954.

29 Koff JL, Shao MX, Ueki IF, et al. Multiple TLRs activate EGFR via a signaling cascade to produce innate immune responses in airway epithelium. Am J Physiol Lung Cell Mol Physiol 2008; 294: L1068-L1075.

30 Verhaeghe C, Tabruyn SP, Oury C, et al. Intrinsic pro-angiogenic status of cystic fibrosis airway epithelial cells. Biochem Biophys Res Commun 2007; 356: 745-749.

31 Machen TE. Innate immune response in CF airway epithelia: hyperinflammatory? Am J Physiol Cell Physiol 2006; 291: C218-C230.

32 Carmeliet P, Jain RK. Molecular mechanisms and clinical applications of angiogenesis. Nature 2011; $473: 298-307$.

33 Lee HS, Myers A, Kim J. Vascular endothelial growth factor drives autocrine epithelial cell proliferation and survival in chronic rhinosinusitis with nasal polyposis. Am J Respir Crit Care Med 2009; 180: 1056-1067.

34 Hoshino M, Takahashi M, Aoike N. Expression of vascular endothelial growth factor, basic fibroblast growth factor, and angiogenin immunoreactivity in asthmatic airways and its relationship to angiogenesis. J Allergy Clin Immunol 2001; 107: 295-301.

35 Wilke M, Buijs-Offerman RM, Aarbiou J, et al. Mouse models of cystic fibrosis: phenotypic analysis and research applications. J Cyst Fibrosis 2011; 10: Suppl. 2, S152-S171.

36 Kim SR, Lee KS, Park HS, et al. HIF-1 $\alpha$ inhibition ameliorates an allergic airway disease via VEGF suppression in bronchial epithelium. Eur J Immunol 2010; 40: 2858-2869.

37 Tyner JW, Kim EY, Ide K, et al. Blocking airway mucous cell metaplasia by inhibiting EGFR antiapoptosis and IL13 transdifferentiation signals. J Clin Invest 2006; 116: 309-321.

38 Takeyama K, Fahy JV, Nadel JA. Relationship of epidermal growth factor receptors to goblet cell production in human bronchi. Am J Respir Crit Care Med, 163: 511-516.

39 Flume PA, Yankaskas JR, Ebeling M, et al. Massive hemoptysis in cystic fibrosis. Chest 2005; 128: 729-738.

40 Ramsey BW, Davies J, McElvaney NG, et al. A CFTR potentiator in patients with cystic fibrosis and the G551D mutation. N Engl J Med 2011; 365: 1663-1672.

41 Rogan MP, Stoltz DA, Hornick DB. Cystic fibrosis transmembrane conductance regulator intracellular processing, trafficking, and opportunities for mutation-specific treatment. Chest 2011; 139: 1480-1490. 